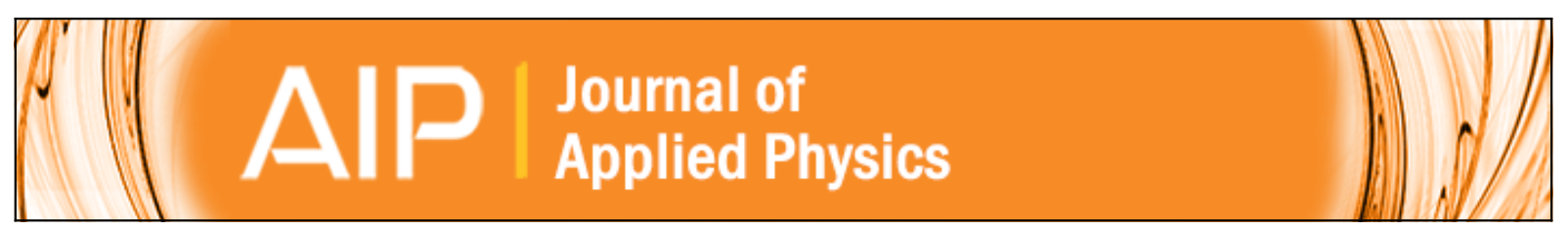

\title{
On the modified active region design of interband cascade lasers
}

M. Motyka, K. Ryczko, M. Dyksik, G. Sęk, J. Misiewicz, R. Weih, M. Dallner, S. Höfling, and M. Kamp

Citation: Journal of Applied Physics 117, 084312 (2015); doi: 10.1063/1.4913391

View online: http://dx.doi.org/10.1063/1.4913391

View Table of Contents: http://scitation.aip.org/content/aip/journal/jap/117/8?ver=pdfcov

Published by the AIP Publishing

\section{Articles you may be interested in}

Eight-band $k \cdot p$ modeling of InAs/lnGaAsSb type-II W-design quantum well structures for interband cascade lasers emitting in a broad range of mid infrared

J. Appl. Phys. 114, 223519 (2013); 10.1063/1.4843076

onHigh-peak-power strain-compensated GalnAs/AllnAs quantum cascade lasers $\left(\begin{array}{lll}\lambda \sim 6 & \mu \mathrm{m}\end{array}\right)$ based on a slightly diagonal active region design

Appl. Phys. Lett. 93, 251110 (2008); 10.1063/1.3054165

Mid-infrared In As/Al Ga Sb superlattice quantum-cascade lasers

Appl. Phys. Lett. 87, 211113 (2005); 10.1063/1.2136428

Effects of anisotropic $k \cdot p$ interactions on energy bands and optical properties of type-II interband cascade lasers J. Appl. Phys. 96, 1866 (2004); 10.1063/1.1763243

Theoretical investigation of mid-infrared interband cascade lasers based on type II quantum wells J. Appl. Phys. 84, 5357 (1998); 10.1063/1.368789

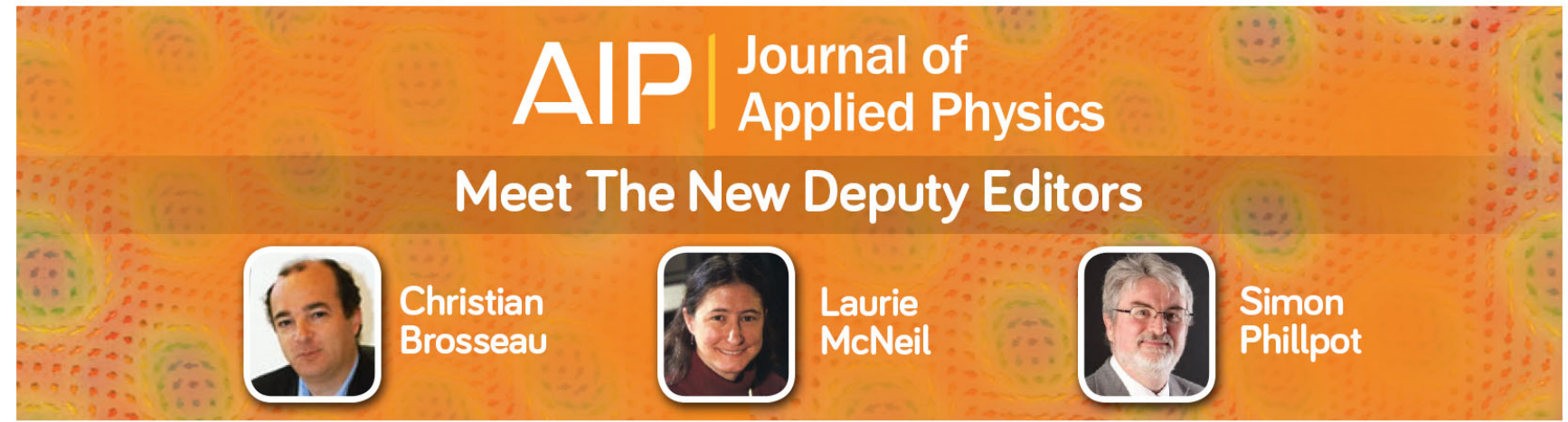




\title{
On the modified active region design of interband cascade lasers
}

\author{
M. Motyka, ${ }^{1}$ K. Ryczko, ${ }^{1}$ M. Dyksik, ${ }^{1}$ G. Sęk, ${ }^{1}$ J. Misiewicz, ${ }^{1}$ R. Weih,${ }^{2}$ M. Dallner, ${ }^{2}$ \\ S. Höfling, ${ }^{2,3}$ and M. Kamp ${ }^{2}$ \\ ${ }^{1}$ Laboratory for Optical Spectroscopy of Nanostructures, Department of Experimental Physics, \\ Wrocław University of Technology, Wybrzeże Wyspiańskiego 27, Wrocław, Poland \\ ${ }^{2}$ Technische Physik, University of Würzburg \& Wilhelm-Conrad-Röntgen-Research Center \\ for Complex Material Systems, Am Hubland, D-97074 Würzburg, Germany \\ ${ }^{3}$ SUPA, School of Physics and Astronomy, University of St. Andrews, North Haugh, KY16 9SS, St. Andrews, \\ United Kingdom
}

(Received 16 November 2014; accepted 11 February 2015; published online 25 February 2015)

\begin{abstract}
Type II InAs/GaInSb quantum wells (QWs) grown on GaSb or InAs substrates and designed to be integrated in the active region of interband cascade lasers (ICLs) emitting in the mid infrared have been investigated. Optical spectroscopy, combined with band structure calculations, has been used to probe their electronic properties. A design with multiple InAs QWs has been compared with the more common double W-shaped QW and it has been demonstrated that it allows red shifting the emission wavelength and enhancing the transition oscillator strength. This can be beneficial for the improvements of the ICLs performances, especially when considering their long-wavelength operation. (C) 2015 AIP Publishing LLC. [http://dx.doi.org/10.1063/1.4913391]
\end{abstract}

\section{INTRODUCTION}

Interband Cascade Lasers (ICLs) based on type II InAs/ GaInSb quantum wells $(\mathrm{QWs})^{1-3}$ have been successfully used for optical detection of many gases related to environmental protection, medical diagnostics, safety and security, in such applications as for instance human breath analysis ${ }^{4}$ or detection of harmful or toxic gases, e.g., formaldehyde ${ }^{5}$ or nitric oxide. ${ }^{6}$ It has been made possible due to the high sensitivity of such laser-based sensors, originating mostly from unique operational characteristics of ICLs, such as, among others: (i) single mode, continuous wave (cw) and high power operation at room or even elevated temperatures, ${ }^{7,8}$ (ii) broad spectral tunability, ${ }^{9}$ (iii) and low threshold currents and small electrical power consumption ${ }^{10}$ when compared to the main competitor, which are quantum cascade lasers (QCLs). However, the best ICLs' performances, when regarding the $\mathrm{cw}$ mono mode lasing at room temperature, have mostly been obtained for the emission range of about 3-5.5 $\mu \mathrm{m}$. There have also been reported some attempts at longer wavelengths, but these are limited to multimode pulsed lasers at above $6 \mu \mathrm{m}$ (Refs. 11 and 12) or low temperature pulsed operation at 7.5 and $10.4 \mu \mathrm{m},{ }^{13,14}$ which are usually insufficient for most of the gas detection schemes. Therefore, there is still a strong demand on improvements in the design and performances of the ICLs to extend the spectral range in which they become suitable for gas sensing applications and could compete with the QCLs.

There already exist reports on new concepts to expand the possible ICL emission range, e.g., by replacing the ternary GaInSb valence band well with a quaternary GaInAsSb layer. ${ }^{15-17}$ This approach offers simultaneous reduction of the strain and a shift of the optical transition to longer wavelengths, while preserving a good electron-hole wave functions overlap. However, this prospective solution has been found out to have some technological limitations related to interface quality and nonradiative centers ${ }^{16}$ and lasers of that kind have not been demonstrated yet.

In this work, we explore another optimization concept which is based on the use of additional layers in the QW structure in comparison to the standard "W-shape" design. We consider a system of triple InAs layers and double GaInSb layers - see Fig. 1, which has already been recognized as very useful from the point of view of wider tunability of the ICLs. ${ }^{9}$ Hereby, we pay attention to the enhancement of the oscillator strength (OS), potentially beneficial for improvements in the performances of the existing devices or for the extension of the emission wavelength. Shifting the emission into longer wavelengths denotes naturally a decrease in the OS, if keeping the common design and materials. In order to investigate this issue, two pairs of double and triple QWs have been grown on both GaSb and InAs substrates. Their electronic structure has been calculated within the multiband $\mathrm{k} \cdot \mathrm{p}$ model and optical spectroscopy in the form of modulated reflectivity has been utilized to probe the optical transition energies and intensities experimentally, and thus to verify the theoretical predictions.

\section{EXPERIMENTAL}

The investigated structures have been grown on (100) oriented InAs (samples A, B) and GaSb (samples C, D) substrates, in a solid source molecular beam epitaxy system equipped with valved cracker cells for both antimony and arsenic. Two of the type II samples have been designed in the "W-like" shape, consisting of two InAs layers and one GaInSb layer (standard double QW design), and two in the form of a triple QW, i.e., three InAs layers and two GaInSb layers-see Fig. 1. All the QW details are listed in Table I.

The core part of all the QWs, as indicated in Table I, is always surrounded by $2 \mathrm{~nm}$ thick AlSb barriers. In order to enhance the overall optical response, the wells have been repeated five times in all the samples. For the $\mathrm{A}$ and $\mathrm{B}$ 


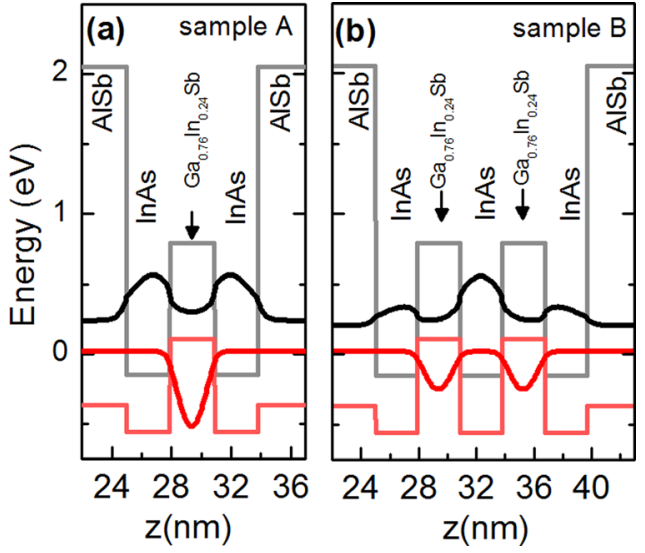

FIG. 1. Band structure diagram of the investigated W-shape double QW (a), and the modified triple QW design (b), together with calculated probability densities of the confined electron and heavy holes states (example for samples A and B).

structures on InAs, the QW part is preceded by $200 \mathrm{~nm}$ InAs buffer. The wells are separated by $25 \mathrm{~nm}$ thick $\mathrm{GaAs}_{0.08} \mathrm{Sb}_{0.92}$ layers lattice matched to InAs, and the entire structure is terminated with the same $\mathrm{GaAs}_{0.08} \mathrm{Sb}_{0.92}$ layer. For the samples $\mathrm{C}$ and $\mathrm{D}$ on GaSb substrate, the active part is similarly constructed, with a difference in the buffer layer being GaSb in this case, and the separating barriers and the cap layer, which are also made of $\mathrm{GaSb}$.

In order to measure photoreflectance (PR) in a wide spectral range, i.e., optical spectra which are sensitive to the transition oscillator strength, ${ }^{15}$ we used a setup based on a Fourier-transformed (FT) and vacuumed Bruker Vertex 80v spectrometer operated in a step- or rapid-scan (for fast differential reflectivity measurements ${ }^{18}$ ) mode. This is additionally equipped with a reflectance/transmittance unit providing $12^{\circ}$ angle for incident probing light, for experiments employing an additional modulated laser beam. Similar FT-based approaches have been demonstrated as very efficient for probing QW transitions in the mid infrared range by several groups. ${ }^{19-22}$ In our case, a liquid-nitrogen cooled MCT (mercury cadmium telluride) photodiode was used as a detector. In this measurement configuration, the pump beam was provided by the $660 \mathrm{~nm}$ line of a semiconductor laser diode, which was mechanically chopped at a frequency of $275 \mathrm{~Hz}$. Phase sensitive detection of the optical response was performed using a lock-in amplifier. Further experimental details can be found elsewhere. ${ }^{21}$

\section{RESULTS AND DISCUSSION}

Figure 1 shows the band diagrams for sample A (left panel) and sample B (right panel), together with the probability

TABLE I. The layer structure of the investigated QWs.

\begin{tabular}{lcc}
\hline \hline Sample: & $\begin{array}{c}\text { InAs (width, } \\
\text { layer numbers) }\end{array}$ & $\begin{array}{c}\text { GaInSb (width, } \\
\text { content, layer numbers) }\end{array}$ \\
\hline Sample A & $2.9 \mathrm{~nm}, 2$ layers & $3 \mathrm{~nm}, \mathrm{Ga}_{0.76} \mathrm{In}_{0.24} \mathrm{Sb}$ layer, 1 layer \\
Sample B & $2.9 \mathrm{~nm}, 3$ layers & $3 \mathrm{~nm}, \mathrm{Ga}_{0.76} \mathrm{In}_{0.24} \mathrm{Sb}$ layer, 2 layers \\
Sample C & $1.4 \mathrm{~nm}, 2$ layers & $3.5 \mathrm{~nm}, \mathrm{Ga}_{0.665} \mathrm{In}_{0.335} \mathrm{Sb}$ layer, 1 layer \\
Sample D & $1.4 \mathrm{~nm}, 3$ layers & $3.5 \mathrm{~nm}, \mathrm{Ga}_{0.665} \mathrm{In}_{0.335}$ Sb layer, 2 layers \\
\hline \hline
\end{tabular}

densities of the first confined electron state (black curve) and the first confined heavy hole state (red curve). It visualizes the expected increase in the overlap between the electron and hole wave functions when increasing the number of potential wells. The situation looks qualitatively very similar for samples $\mathrm{C}$ and D. The overlap integral change translates directly into the change of the optical transition oscillator strength defined as

$$
f \sim \frac{P_{i f}^{2}}{E^{f}-E^{i}}\left|\left\langle F_{f}(z) \mid F_{i}(z)\right\rangle\right|^{2},
$$

where $P_{i f}$ is the bulk momentum matrix element defined basically by the Kane energy, whereas $\left|\left\langle F_{f}(z) \mid F_{i}(z)\right\rangle\right|$ is the so called envelope wave function overlap integral.

Figure 2 shows photoreflectance spectra obtained for all the four investigated samples. The red solid line curves represent the result of the fitting procedure assuming the PR line-shape according to the commonly used formula ${ }^{15,23,24}$

$$
\frac{\Delta R}{R}(E)=\operatorname{Re}\left[\sum_{j=1}^{n} C_{j} \cdot e^{i \cdot \vartheta_{j}}\left(E-E_{j}+i \cdot \Gamma_{j}\right)^{-m_{j}}\right],
$$

where $n$ is the number of optical transitions and independent spectral functions used in the fit, $C_{j}$ and $\vartheta_{j}$ are the amplitude and phase parameters, $E_{j}$ and $\Gamma_{j}$ are the energy and the broadening parameter of the transitions, respectively. The fitting curves are shown as solid red lines together with the moduli of the individual resonances (dashed lines) obtained according to Eq. (3)

$$
\Delta \rho(E)=\frac{|C|}{\left[\left(E-E_{0}\right)^{2}+\Gamma^{2}\right]^{\frac{m}{2}}} .
$$

First of all, it is visible that the introduction of the additional layers in the QW structure, i.e., expansion into the triple QW

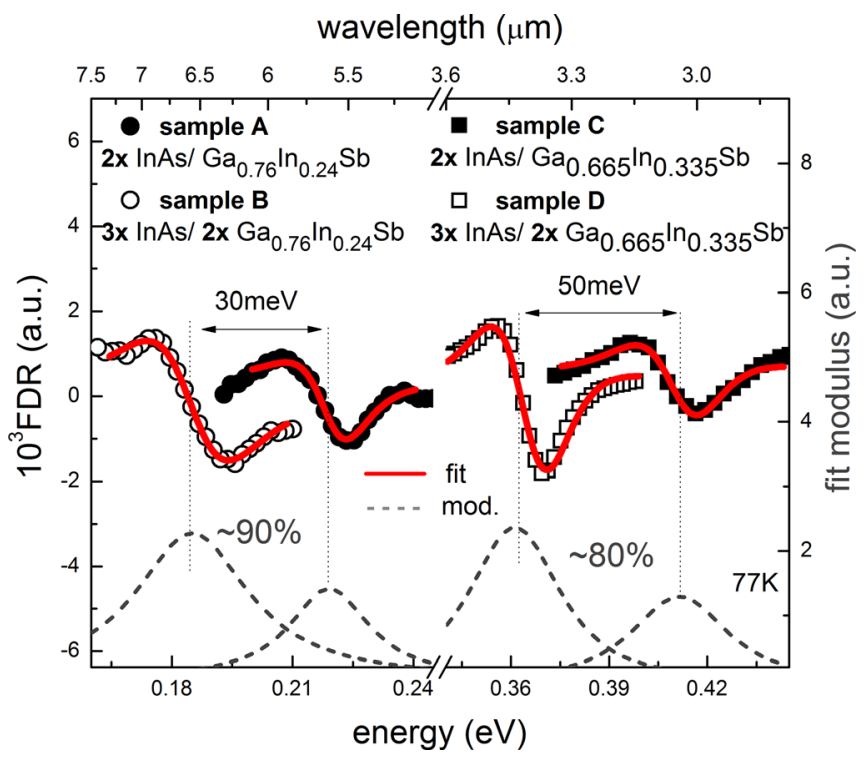

FIG. 2. Low temperature $(77 \mathrm{~K})$ photoreflectance spectra for the investigated samples: A-full circles, B-open circles, C-full squares, and D-open squares. The red solid lines represent the line shape fits, whereas the dashed peaks correspond to the moduli of the respective PR features. 
system, shifts the fundamental transition energy to the red. Such a behavior is expected when taking into account the effectively wider confinement potential for the carriers. When comparing the spectra of samples A and B, and then samples $\mathrm{C}$ and $\mathrm{D}$, energy shifts of $30 \mathrm{meV}$ and $50 \mathrm{meV}$ are obtained, respectively. These translate into wavelength shifts of $\sim 1000 \mathrm{~nm}$ and $\sim 400 \mathrm{~nm}$, respectively. This indicates that such an active region modification can indeed lead to a broader tunability, as already suggested in the recent report, ${ }^{9}$ but mainly it shows that such a change makes the tuning of the emission into the longer wavelengths easier, which can be one of the advantages of this kind of modified triple-QWbased active region design.

Further, we have used the property of the modulated reflectivity spectra, i.e., probing the intensity of the optical transitions, ${ }^{25-27}$ where the integrated spectral feature intensity corresponds to the transition oscillator strength. The former can directly be obtained as an area under the moduli of the particular spectral lines, i.e., under the dashed curves in Fig. 2. It should be mentioned here that transition intensities determined in such a way can be compared between the spectra of different samples only if one can assume that the modulation efficiency is approximately the same. This is the case for the samples investigated here: they have similar layer structure and the experimental conditions have been preserved.

For the two pairs of the samples, we have obtained an increase in the PR intensity by about $90 \%$ and $80 \%$ when changing from the double to the triple $\mathrm{QW}$ design (comparing sample A with $\mathrm{B}$ and then sample $\mathrm{C}$ with $\mathrm{D})$. For further analysis, we assume that the value of $P_{i f}^{2} /\left(E^{f}-E^{i}\right)$ (in Eq. (1)) does not change significantly between various QW samples investigated here. We can also replace the initial and final states with the hole $h$ and electron $e$ states, respectively, and the oscillator strength can be rewritten in the following form:

$$
f \sim\left|\left\langle F_{e}(z) \mid F_{h}(z)\right\rangle\right|^{2}=\left|\int_{-\infty}^{\infty} F_{e}^{*}(z) \cdot F_{h}(z) \mathrm{d} z\right|^{2},
$$

i.e., considered as proportional to the squared wave functions overlap. Hence, this squared overlap will further be treated as an approximate figure of merit of the transition intensity and will serve as a quantity to be confronted with the experiment.

Figure 3 presents the calculated transition energies (panel (a)) and the calculated squared overlap integral of the electron and hole wave functions (panel (b)) as a function of the InAs layer width of the QWs. The calculations have been performed within the eight-band $\mathbf{k} \cdot \mathbf{p}$ formalism ${ }^{28}$ including the strain effect, with all the methodology similar to that described in one of our previous works. ${ }^{17}$ All the material parameters have been taken from Ref. 29 and have been interpolated linearly for the corresponding ternary compounds, except for the band gap. In the case of the band gap determination as a function of alloy composition, we included the respective bowing parameters. ${ }^{29}$ Any additional structural effects occurring for heterostructures of

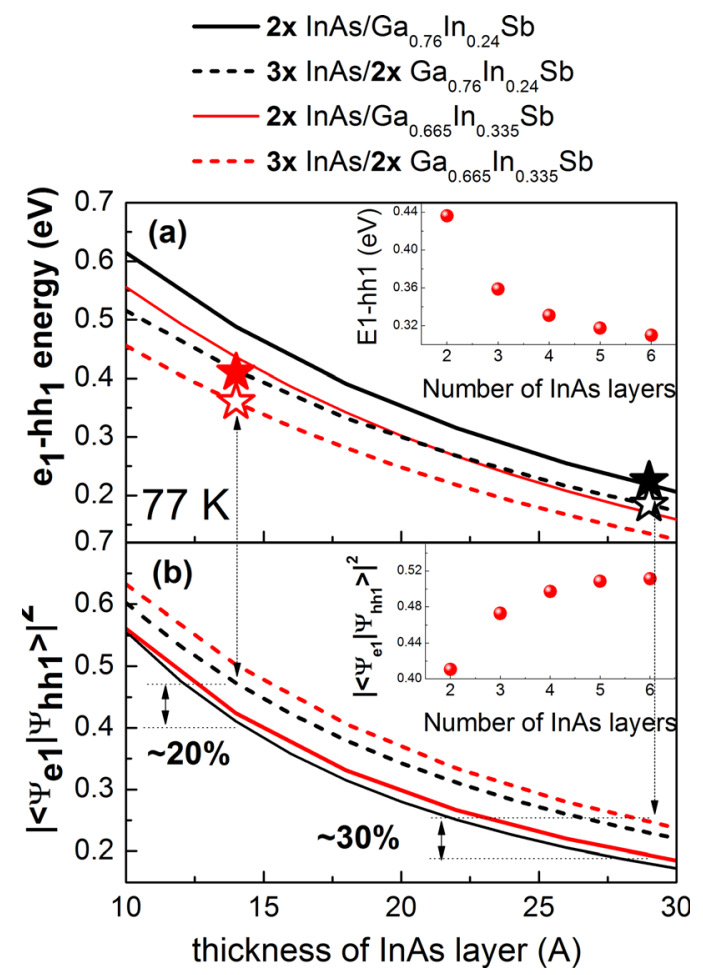

FIG. 3. Calculated optical transition energies-(a), and squared electronhole wave function overlaps-(b); black solid line-sample A; black dashed line_-sample B; red solid line-sample C; red dashed line-sample D. The insets show the dependence of the type II transition energy and squared wave functions overlap as a function of the number of the wells in the system corresponding to the set of samples C and D.

this material combination, as e.g., disturbance of the confinement potential due to the existence of a GaAs-like or InSb-like interfaces ${ }^{30,31}$ nor interface intermixing have not been included in the calculations.

Solid lines in Fig. 3 represent the results of calculations for the samples with the standard "W" shape double QW profile, like sample A (black solid curves) and sample C (red solid curves), whereas the dashed lines correspond to the calculations performed for the modified design structures with three InAs layers: sample B-black dashed lines, and sample D-red dashed lines. Star symbols correspond to the energies of the fundamental transitions obtained from the PR measurements (from spectra shown in Fig. 2) for all the investigated samples. It is seen that the symbols match the calculated dependencies very well. These results show both theoretically and experimentally that the QW multiplication in such a type II system offers the intrinsic property of shifting the optical transitions to the red, with respect to common W-design QWs. Therefore, this can be a useful solution for longer wavelength emission, as long as the transition oscillator strength can be kept sufficiently large.

When considering the overlap integrals, we can compare the solid curves with the dashed ones to observe the QW design modification. In the entire range of the InAs layer widths, we can see an increase in the squared overlap integral of about $30 \%$ and $20 \%$ for sample B (comparing to sample A) and sample D (comparing to sample C), respectively. The calculated enhancement of the squared wave functions overlap integral after introducing additional layers in the 
QW structure (with respect to the standard "W-shaped" QWs) agrees qualitatively with the experimental data reflecting the transition intensities. In spite of the clear and confirmed tendencies observed both in the calculation and in the experiment, the relative numbers concerning the intensities do not agree very exactly. This is however related to some limitations on both the experimental and the theoretical side. In the modulated reflectivity measurements, there always remains a certain inaccuracy related to the proper alignment of the probe and pump beam spots on the surface of the sample, and hence keeping exactly the same photo-modulation efficiency for each sample is difficult. On the other hand, when regarding the calculations, the QW layout is assumed the simplest possible and such issues as the band bending at the interfaces, the interface intermixing nor the existence of any intermediate interfacial layers have not been taken into account.

The insets in Fig. 3 show how both the transition energy and its intensity would change with further increase in the number of wells (layers) in this kind of QW system for the case corresponding to the previously discussed samples $\mathrm{C}$ and D. As expected, the dependencies start saturating and although the possible use of a fourfold repetition of the InAs QW can give an improvement in the oscillator strength by several percent, so it is still perhaps worth consideration in the practical realization, further switching into five wells enhances the transition intensity rather negligibly. The dependence on the number of wells in the system looks qualitatively very similar also for samples A and B; therefore, it is not shown in Fig. 3 for clarity.

In order to predict the usefulness of the discussed approach and the OS enhancement on the full laser structure, in which the active region is in an external electric field, simulations taking that effect into account have also been performed. Figure 4 shows the calculated fundamental transition energy (top panel) and the squared wave functions overlap (bottom panel) for the typical "W" QW (solid lines) and the triple design QW (dashed lines) under the external electric field equal to $100 \mathrm{kV} / \mathrm{cm}$ (a value characteristic for the ICL devices). The results are plotted as a function of parameter "d," which is the thickness of the one (or two) InAs layers in the case of the double (triple) QW designs. The entire layer structure must be modified accordingly in the electric field in order to compensate its influence, mainly on the oscillator strength. As the electric field bends the structure, the electron and hole probability densities are shifted in the opposite directions, which leads to the overlap reduction (and simultaneous shift of the transition energy). This can be balanced if the structure is made asymmetric. For the common W-shape double QW design, it is sufficient to just change the thickness of one of the InAs layers (the tuned layer thickness is named "d" in Fig. 4). The situation is much more complex in the case of the triple QW design. First of all, there need to be modified at least two of the three InAs wells to effectively push back the electron probability density and compensate the electric field influence. Second, as the holes are confined in two GaInSb layers now, this must be corrected by changing the thickness of one of these layers as well. Exemplary results of such modifications are

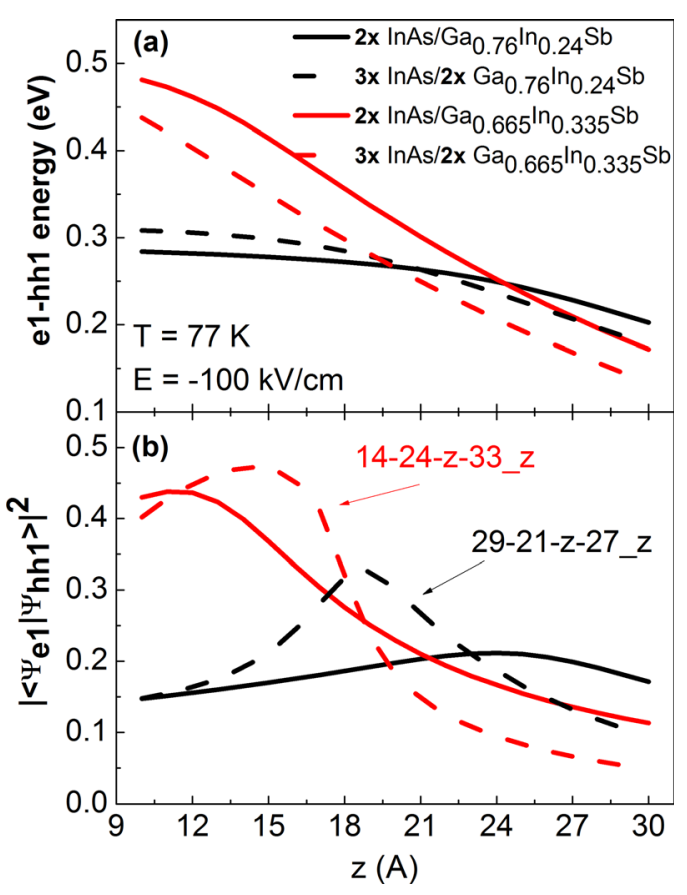

FIG. 4. Calculated transition energy-(a), and squared wave functions overlap integral-(b) versus the thickness of the InAs layers (labeled "d") for the double W-shaped QWs (black lines) and the upgraded triple QW design (red lines), in the conditions imitating the device structure, i.e., in external electric field of $100 \mathrm{kV} / \mathrm{cm}$.

shown in Fig. 4 with the dashed lines. The widths of the layers in the triple QW structure are given in the figure. Of course, one could propose other design corrections, but disregarding the details the main conclusion is that it is always possible to maximize the oscillator strength of the transitions in such a multiple type II QW system in the electric field, and that the structure with triple InAs QWs always offers larger values of the OS. Therefore, this also further proves the usefulness of this multiplied QW approach.

It is also worth mentioning that in order to verify preliminarily the effect of the increased number of wells in the active region such a modification has been included in the simulations of the optical field distribution within the waveguide and the resulting confinement factor. The obtained modal overlap with the active region has appeared to be higher for the triple QW design in spite of the slightly wider active region. This is related to the larger refractive index of GaInSb layers, which tends to "concentrate" the optical mode at the core of the structure. Thus, the thicker active region has in fact a positive effect on the confinement of the mode, and therefore, when combined with the larger oscillator strength of the active transitions, the triple QW design can indeed improve at least some of the ICL characteristics.

\section{CONCLUSION}

In conclusion, it has been shown that the modification of the standard "W" shape of QWs by introducing additional InAs and GaInSb layers allows enhancing the OS of the type II optical transition and simultaneously red shifts the emission. These properties have been verified experimentally by means of modulation spectroscopy and demonstrated for two 
types of structures, one grown on a $\mathrm{GaSb}$ substrate and designed for shorter wavelength of about $4 \mu \mathrm{m}$, but also the second one grown on an InAs substrate, with the emission in the 6-7 $\mu \mathrm{m}$ range. The mentioned benefits have additionally been supported by simulating the effect of the external electric field and imitating the conditions occurring in operational devices. The electric field effect on the wave functions and their overlaps can be compensated with the structure asymmetry, which still allows gaining in the optical transition intensity for the triple QW design. Eventually, we showed that increasing the number of layers in this kind of a type II system can give some improvements yet when four InAs QW layers are considered, whereas a larger number of layers cause the transition energy and oscillator strength to be saturated and almost insensitive to the structure change. Summarizing, we have shown that such a multiplication of the type II QW structure can be a way to develop ICL devices with improved performances and perhaps able to compete with the existing solutions also in the longer wavelength mid-infrared, significantly beyond $5 \mu \mathrm{m}$.

\section{ACKNOWLEDGMENTS}

The work has been supported by Project Widelase (No. 318798) of the 7-th Framework Program of the European Commission. We would like to acknowledge the National Science Centre of Poland for support within Grant No. 2011/ 03/D/ST3/02640.

${ }^{1}$ R. Q. Yang, Superlattices Microstruct. 17, 77-83 (1995).

${ }^{2}$ J. R. Meyer, C. A. Hoffman, F. J. Bartoli, and L. R. Ram-Mohan, Appl. Phys. Lett. 67, 757 (1995).

${ }^{3}$ A. Bauer, F. Langer, M. Dallner, M. Kamp, M. Motyka, G. Sek, K. Ryczko, J. Misiewicz, S. Höfling, and A. Forchel, Appl. Phys. Lett. 95, 251103 (2009).

${ }^{4}$ T. H. Risby and F. K. Tittel, Opt. Eng. 49, 111123 (2010).

${ }^{5}$ S. Lundqvist, P. Kluczynski, R. Weih, M. v. Edlinger, L. Nähle, M. Fischer, A. Bauer, S. Höfling, and J. Koeth, Appl. Opt. 51, 6009-6013 (2012).

${ }^{6}$ M. von Edlinger, J. Scheuermann, R. Weih, C. Zimmermann, L. Nähle, M. Fischer, J. Koeth, and S. Höfling, IEEE Photonics Technol. Lett. 26, 480 (2014).
${ }^{7}$ W. W. Bewley, C. S. Kim, C. L. Canedy, C. D. Merritt, I. Vurgaftman, J. Abell, J. R. Meyer, and M. Kim, Appl. Phys. Lett. 103, 111111 (2013).

${ }^{8}$ C. S. Kim, M. Kim, J. Abell, W. W. Bewley, C. D. Merritt, C. L. Canedy, I. Vurgaftman, and J. R. Meyer, Appl. Phys. Lett. 101, 061104 (2012).

${ }^{9}$ Y. Jiang, L. Li, Z. Tian, H. Ye, L. Zhao, R. Q. Yang, T. D. Mishima, M. B. Santos, M. B. Johnson, and K. Mansour, J. Appl. Phys. 115, 113101 (2014).

${ }^{10}$ I. Vurgaftman, W. W. Bewley, C. L. Canedy, C. S. Kim, M. Kim, C. D. Merritt, J. Abell, J. R. Lindle, and J. R. Meyer, Nature Commun. 2, 585 (2011).

${ }^{11}$ M. Dallner, S. Höfling, and M. Kamp, Electron. Lett. 49, 286 (2013).

${ }^{12}$ L. Li, L. Zhao, Y. Jiang, R. Q. Yang, J. C. Keay, T. D. Mishima, M. B. Santos, and M. B. Johnson, Appl. Phys. Lett. 101, 171118 (2012).

${ }^{13}$ Z. Tian, R. Q. Yang, T. D. Mishima, M. B. Santos, and M. B. Johnson, IEEE Photonics Technol. Lett. 21, 1588 (2009).

${ }^{14}$ Z. Tian, L. Li, H. Ye, R. Q. Yang, T. D. Mishima, M. B. Santos, and M. B. Johnson, Electron. Lett. 48, 113 (2012).

${ }^{15}$ F. Janiak, G. Sek, M. Motyka, K. Ryczko, J. Misiewicz, A. Bauer, S. Höfling, M. Kamp, and A. Forchel, Appl. Phys. Lett. 100, 231908 (2012).

${ }^{16}$ F. Janiak, M. Motyka, G. Sek, M. Dyksik, K. Ryczko, J. Misiewicz, R. Weih, S. Höfling, and M. Kamp, J. Appl. Phys. 114, 223510 (2013).

${ }^{17}$ K. Ryczko, G. Sẹk, and J. Misiewicz, J. Appl. Phys. 114, 223519 (2013).

${ }^{18}$ M. Motyka and J. Misiewicz, Appl. Phys. Express 3, 112401 (2010).

${ }^{19}$ T. J. C. Hosea, M. Merrick, and B. N. Murdin, Phys. Status Solidi A 202, 1233 (2005).

${ }^{20}$ J. Shao, W. Lu, F. Yue, X. Lu, W. Huang, Z. Li, and S. Guo, Rev. Sci. Instrum. 78, 013111 (2007).

${ }^{21}$ M. Motyka, G. Sęk, F. Janiak, J. Misiewicz, K. Kłos, and J. Piotrowski, Meas. Sci. Technol. 22, 125601 (2011).

${ }^{22}$ D. D. Firsov and O. S. Komkov, Tech. Phys. Lett. 39, 1071-1073 (2013).

${ }^{23}$ F. H. Pollak, "Modulation spectroscopy of semiconductors and semiconductor microstructures," in Handbook on Semiconductors, edited by T. S. Moss (Elsevier Science, Amsterdam, 1994), Vol. 2, pp. 527-635.

${ }^{24}$ D. E. Aspnes, Surf. Sci. 37, 418 (1973).

${ }^{25}$ R. Kudrawiec, G. Sek, K. Ryczko, J. Misiewicz, and J. C. Harmand, Appl. Phys. Lett. 84, 3453 (2004).

${ }^{26}$ S. Turcotte, S. Larouche, J.-N. Beaudry, L. Martinu, R. A. Masut, P. Desjardins, and R. Leonelli, Phys. Rev. B 80, 085203 (2009).

${ }^{27}$ W. Rudno-Rudziński, K. Ryczko, G. Sẹk, J Misiewicz, E. S. Semenova, A. Lemaitre, and A. Ramdane, Semicond. Sci. Technol. 26, 085004 (2011).

${ }^{28}$ S. Ridene, K. Boujdaria, H. Bouchriha, and G. Fishman, Phys. Rev. B 64, 085329 (2001).

${ }^{29}$ I. Vurgaftman and J. R. Meyer, J. Appl. Phys. 94, 3675 (2003).

${ }^{30}$ A. Jasik, I. Sankowska, D. Pierścińska, K. Regiński, K. Pierściński, and J. Kubacka-Traczyk, J. Appl. Phys. 110, 123103 (2011).

${ }^{31}$ X. Junliang, Z. Yu, L. Yongping, W. Juan, X. Wei, H. Hongyue, X. Yingqiang, and N. Zhichuan, J. Appl. Phys. 116, 123107 (2014). 